\title{
A Rare Cause of Acute Abdomen in a 59-Year-Old Male
}

\author{
Ayrton Bangolo Sarah Pender Chandini Sajja Mahabuba Akhter \\ Swati Govil Abraham Lo \\ Department of Internal Medicine, Hackensack Meridian Health/Palisades Medical Center, \\ North Bergen, NJ, USA
}

\section{Keywords}

Celiac artery dissection · Aortic dissection · Acute abdomen · Differential diagnosis

\begin{abstract}
Celiac artery dissection is often an inconspicuous cause of acute abdomen. Spontaneous celiac artery dissection is most often associated with aortic dissection, and thus isolated spontaneous celiac artery dissection is rare. Herein, we present a case of celiac artery dissection with no aortic involvement. This case emphasizes the importance of including such a condition in the differential diagnosis of patients presenting with acute abdomen.
\end{abstract}

(c) 2021 The Author(s).

Published by S. Karger AG, Basel

\section{Introduction}

Spontaneous celiac artery dissection is a rare phenomenon as most cases of celiac artery dissection are iatrogenic secondary to surgery [1]. Spontaneous celiac artery dissection is most often not isolated but associated with aortic artery dissection due to the celiac axis arising from the abdominal aorta [1]. Celiac artery dissection may also propagate into the surrounding vessels such as the left gastric, splenic, and common hepatic arteries [2]. Involvement of the hepatic artery can present with abdominal pain and elevated liver function tests [2]. Here, we report an unusual case of acute abdomen in a patient found to have spontaneous celiac artery dissection without aortic involvement. This case illustrates the importance of incorporating such a condition in the differential diagnosis of patients presenting with acute abdomen.

\section{Karger!}




\section{Case Presentation}

This case is a 59-year-old male with a past medical history significant for diabetes mellitus and hypertension who presented with a 1-day history of epigastric pain. This insidious onset of pain was constant and "throbbing" in character, radiating to the left and right upper quadrants. Associated symptoms included a few episodes of nonbloody and watery diarrhea. of note, the patient had a cholecystectomy 3 months prior and tonsillectomy 1 week prior to presentation. On physical examination, the patient was hypertensive without pulse deficit (femoral pulse was present and equal bilaterally). There was generalized abdominal tenderness localized more so to the epigastric region. Laboratory values were significant for aspartate aminotransferase 57 units/L (13-39), alanine transaminase 195 units/L (7-52), alkaline phosphatase 206 units/L (34-104), lactate $3 \mathrm{mmol} / \mathrm{L}(<2)$, and lipase 24 units/L (11-82). CT abdomen/pelvis with intravenous (IV) contrast revealed a circumferential mural hypodensity along the celiac artery, extending into the adjacent splenic and hepatic arteries; the appearance was suggestive of celiac artery dissection (Fig. 1). Heparin continuous infusion was then initiated.

Due to bleeding in the oropharynx, heparin continuous infusion was later discontinued. An acute hepatitis panel workup was negative, and right upper quadrant ultrasound revealed echogenic liver representing fatty infiltration. Repeat CT abdomen/pelvis with IV contrast on day 2 showed stable celiac artery dissection with intramural hematoma. After 4 days of hospitalization, the patient's abdominal pain had resolved, and he was able to tolerate regular diet. He was discharged with aspirin and his home antihypertensive medications with instructions for repeat CT abdomen/pelvis with IV contrast in 6 months.

\section{Discussion/Conclusion}

One must consider isolated spontaneous celiac artery dissection in the differential diagnosis for acute abdomen. Patients with isolated celiac artery dissection may present with the medical history (hypertension and genetics), signs, and symptoms consistent with abdominal aortic dissection but lack the physical exam findings of pulse deficit seen in up to $30 \%$ of patients with thoracoabdominal aortic dissection [3]. Additionally, liver function test derangements may provide clues of dissection extension from the celiac artery to the hepatic artery [4]. In a case series, vascular complications such as propagation of dissection into surrounding vessels lead to splenic and renal infarcts or hepatic artery aneurysm [2]. Once isolated spontaneous celiac artery dissection is considered, abdominal CT with IV contrast is the gold standard, but other modalities such as magnetic resonance angiography, sonography, or conventional angiography can also be used [2].

The treatment strategy of celiac artery dissection consists of blood pressure control to prevent propagation of the dissection and anticoagulation to prevent thromboembolic complications [5]. Long-term therapy to prevent thromboembolic complications includes anticoagulation with target INR 2.0-3.0 and antiplatelets for 3-6 months [5]. Our patient was started on heparin given the extension of the dissection to the adjacent vessels, but it had to be discontinued given bleeding complications. He was also started on antiplatelet therapy with low-dose aspirin, although he will probably need aspirin lifelong as he is at intermediate risk for cardiovascular events in the next 10 years.

Spontaneous resolution of symptoms is common in isolated spontaneous celiac artery dissection, although some patients may require celiac artery stenting or surgical repair as a result of continued abdominal pain or aneurysmal degeneration [6]. Clinical evaluation

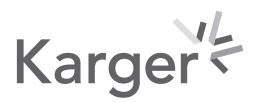


Fig. 1. Initial CT of the abdomen and pelvis with IV contrast, illustrating circumferential mural hypodensity along the celiac artery (red arrow), extending into the adjacent splenic and hepatic arteries, suggestive of celiac artery dissection.

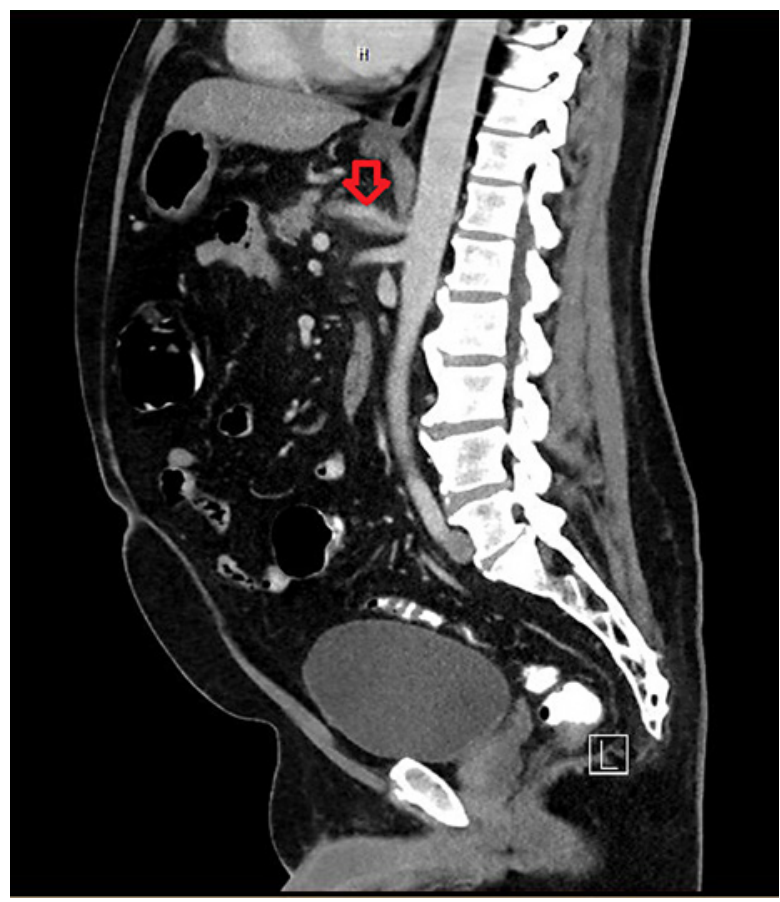

and CT abdomen with IV contrast is recommended at 6 months and 12 months after initial diagnosis, followed by annual duplex ultrasound for 5 years. In symptomatic patients, screening with CT abdomen with IV contrast is continued every 6 months until symptoms resolve [6].

This case of spontaneous celiac artery dissection aims to emphasize the importance of including this rare condition in the differential diagnosis of acute abdomen, especially in middle-aged patients with comorbidities predisposing to vascular disease. Timely antithrombotic therapy can help reduce the risk of thromboembolic complications. Interval imaging will help monitor the dissection expansion as well as aneurysmal degeneration, and actions should be taken accordingly.

\section{Acknowledgments}

This manuscript does not include any nonauthor contributors to acknowledge.

\section{Statement of Ethics}

Written informed consent was obtained from the patient for publication of this case report and any accompanying images. This study protocol was reviewed, and the need for approval was waived by the Ethics Committee at Palisades Medical Center Hackensack Meridian Health.

\section{Conflict of Interest Statement}

No potential conflicts of interest were reported by the authors.

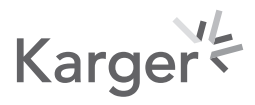




\section{Funding Sources}

No funding was received.

\section{Author Contributions}

Ayrton Bangolo searched the literature and wrote and revised the manuscript. Sarah Pender, Chandini Sajja, Mahabuba Akhter, and Swati Govil revised and edited the manuscript. Abraham Lo revised and approved the final version and is the article's guarantor. All authors certify that they contributed sufficiently to the intellectual content and data analysis. Each author has reviewed the final version of the manuscript and approved it for publication.

\section{Data Availability Statement}

Data sharing is not applicable to this article as no datasets were generated or analyzed during the current study.

\section{References}

1 Gilani R. Spontaneous mesenteric arterial dissection. UpToDate. 2020.

2 D’Ambrosio N, Friedman B, Siegel D, Katz D, Newatia A, Hines J. Spontaneous isolated dissection of the celiac artery: CT findings in adults. AJR Am J Roentgenol. 2007;188:W506-11.

3 Black JH, Manning WJ. Clinical features and diagnosis of acute aortic dissection. UpToDate. 2021.

4 Su KY, Stanhope ML, Kaufman BP. Spontaneous hepatic artery dissection-a rare presentation of fibromuscular dysplasia. Oxf Med Case Reports. 2016;2016(11):omw083.

5 Vaidya S, Dighe M. Spontaneous celiac artery dissection and its management. J Radiol Case Rep. 2010;4(4): 30-3.

6 DiMusto PD, Oberdoerster MM, Criado E. Isolated celiac artery dissection. J Vasc Surg. 2015;61(4):972-6. 